\title{
A new approach assessing the quality of whole slide images by discovering specific issues
}

\section{Yuchen Song}

South China University of Technology

Ying Gao ( $\sim$ gaoying@scut.edu.cn )

South China University of Technology

Changhong Liang

Guangdong General Hospital

\section{Zaiyi Liu}

Guangdong General Hospital

\section{Research}

Keywords: Whole slide images (WSI), Digital pathology, Quality assessment

Posted Date: March 31st, 2020

DOl: https://doi.org/10.21203/rs.3.rs-20180/v1

License: (c) (i) This work is licensed under a Creative Commons Attribution 4.0 International License. Read Full License 
1 A new approach assessing the quality of whole slide images(WSIs) by discovering

2 specific issues

3

4 Yuchen Song ${ }^{1}$, Ying Gao ${ }^{1, *}$, Changhong Liang ${ }^{2}$, Zaiyi Liu $^{2}$

5

$6{ }^{1}$ School of Computer Science and Engineering, South China University of Technology,

7 Guangzhou, Guangdong 510006, China

$8 \quad{ }^{2}$ Department of Radiology, Guangdong Provincial People's Hospital, Guangdong Academy of

9 Medical Sciences, Guangzhou, Guangdong 510080, China

11 *Corresponding Author

12 Y. Gao, School of Computer Science and Engineering, South China University of

13 Technology, Guangzhou 510632, China (E-mail: gaoying@scut.edu.cn).

15 Abstract

16 Background: Since whole slide images (WSIs) are widely used in clinical diagnosis,

17 assessing the quality of these images has generated considerable interest. We divided the

18 evaluation process into different sections focusing on specific issues and developed an

19 approach based on computer technology to assess the quality of WSIs and provide details

20 corresponding to specific issues.

21 Methods: We focused on three issues in this work: position deviation, cover slip

22 misplacement and wrong focus. Our approach revolves around these issues. We collected two 
23 datasets from Guangdong Provincial People's Hospital to develop the approach, discovered

24 three issues and provided details corresponding to these issues.

25 Results: We designed experiments based on two datasets to validate the effectiveness of the

26 approach. The experiments show that using our approach, existing issues can be discovered

27 and corresponding details are provided at a high accuracy. In the training set, the accuracy of

28 the discovered three issues reaches $0.998,0.987$ and 0.988 . In the validation set, the accuracy

29 of discovering all issues reaches 0.914.

30 Conclusions: We proposed an approach focusing on the following specific image issues:

31 position deviation, cover slip misplacement and wrong focus. Using this approach, issues

32 were discovered with high accuracy, and reports were produced that provide details regarding

33 existing issues discovered by our approach. However, a dataset of WSIs with more issues,

34 including stain concentration and poor microtomy, should be established, and methods

35 discovering these issues must be developed in the future.

37 Keywords: Whole slide images (WSI), Digital pathology, Quality assessment

\section{Background}

40 Although pathology is considered the gold standard of clinical diagnosis, the use of

41 microscopy has limitations in that microslides are difficult to store, archive and share. The

42 newly developed whole slide image (WSI) is proposed to solve these limitations. WSIs can

43 digitalize microslide information. In other words, we could use computer technology for

44 clinical applications. Therefore, WSIs are widely used in clinical diagnosis [1-4]. To 
guarantee the accuracy and efficacy of WSIs in place of the direct observation with a

46 microscope, assessing the quality of WSIs has generated considerable interest [5-8]. Initial attempts have focused on assessing the clarity of WSIs. The pathologist's opinion on image quality is the most straightforward choice. Nevertheless, the difference

49 between pathologists in this work results in stability issues due to subjectivity. Furthermore, 50 owing to the massive number required to be evaluated, this method is time-consuming for 51 pathologists [7]. Consequently, automated methods for assessing image quality should be 52 proposed. Walkowski and Szymas [9] showed that the gray level co-occurrence matrix 53 (GLCM) could well represent the sharpness of WSIs. Hashimoto et al. [10] added noise into 54 the analysis model to assess image quality. Other studies have made great effort using different features based on computer technology to represent the quality of WSIs [11-14]. In addition, several tools have been developed to assess image quality automatically. Ameisen et al. [15] developed a method that integrates features including saturation, contrast, and brightness and then used a picture to display the distribution of blurred areas. Janowczyk et al. [16] developed an open source tool to calculate image metrics and identify artifact-free

60 regions with supervised classifiers. Previous studies demonstrated the effectiveness of methods based on computer technology. However, one common issue of the methods mentioned above is that they fail to

63 address the fact that the clarity is not the unique standard to measure image quality. Even 64 though it is simple for a person to evaluate a single WSI by providing a numeric score or a 65 simple description of the quality, such as good or poor, it is a complex task for computer66 based methods. In addition, a numeric score or a simple description of the quality is not 
67 meaningful enough compared to providing information regarding specific issues in WSIs.

68 Hence, we divide the evaluation process into different sections focusing on different specific

69 issues and develop an approach based on computer technology to not only judge the varieties

70 of issues that exist in WSIs but also provide the details corresponding to specific issues.

\section{Methods}

73 The most essential idea in this work is to divide the evaluation process into sections focusing on specific issues. Each section provides a result that contains whether a corresponding issue exists and details related to the issue. These results are integrated into a report that provides a reference to evaluate and enhance the quality of a single WSI. We claim three issues in this work: position deviation, cover slip misplacement and wrong focus [17]. Position deviation means that the scanner failed to obtain complete information due to the deviation between the virtual position and real position on a slide. Cover slip misplacement means that a black line exists and leads to a serious blur problem around the black line. Wrong focus means the

81 failure of obtaining glass slides' texture information due to the wrong focus setting. Figure 1

82 shows an example of the 3 varieties of issues. Our approach addresses these three issues.

\section{Datasets}

85 We collected two datasets in this study. Both datasets are from Guangdong Provincial

86 People's Hospital. The first dataset is composed of 276 gastric cancer WSIs, including 90

87 normal WSIs and 186 WSIs with abnormal quality issues. This dataset is used for training. 88 Actually, multiple types of issues may exist in a WSI. In the training process, each WSI is 
89 labeled by its most apparent issue. A total of 186 WSIs with abnormal quality issues consisted

90 of 74 with position deviation, 38 with cover slip misplacement and 74 with wrong focus. The

91 second dataset is composed of 58 colorectal cancer samples. This dataset is used for

92 validation. As described above, the aim of this work is to generate result reports of issue

93 details. Therefore, WSIs in the validation set are not divided into specific issue types.

94 Datasets are defined as follows:

$95 I_{\text {all }}=\left\{I_{\text {train }}, I_{\text {valid }}\right\}$

$96 \quad I_{\text {train }}=\left\{I_{P D}, I_{C S M}, I_{W F}, I_{N}\right\}$

97 where $I_{\text {train }}$ represents the first dataset and $I_{\text {valid }}$ represents the second dataset. In $I_{\text {train }}$, 98 we use the first letter of each word to represent the problem. For instance, PD represents a 99 position deviation issue.

100

101 Position Deviation

102 It could be easily observed that if a WSI has position deviation, at least one edge has tiles

103 containing useful information for clinical diagnosis. Thus, the number of tiles with clinical

104 information on one edge can represent whether position deviation exists on such edges. With

105 the purpose of calculation, the following steps are required:

1061 We trained a deep convolutional network to judge whether tiles contain useful

107 information for clinical diagnosis.

108 2. We collected tiles on the four edges of WSIs from $I_{N}$ and $I_{P D}$.

109 3. We calculated the count vector of each WSI and selected a suitable threshold to judge 110 whether the WSI has a position deviation issue. 
111 In the first step, our aim is to gain a model that can identify whether tiles split from

112 the WSIs contain useful information for clinical diagnosis. We selected fifteen WSIs and split

113 them into tiles using Openslide [18]. The tiles we collected for training this classifier model

114 are $256 \times 256$ in size at $20 \times$ magnification. We chose samples from the split tiles and trained a

115 deep convolutional network [19] to judge whether tiles contain useful information for clinical

116 diagnosis.

117 Position deviation may exist in more than 1 orientation. Hence, we need to calculate a

118 vector consisting of four values to represent whether position deviation exists and to

119 determine its orientation. In our experiments, we used this method on all WSIs from $I_{N}$ and

$120 I_{P D}$ to acquire the vectors mentioned above. Tiles we collected on edges are $64 \times 64$ in size at

$12110 \times$ magnification. Finally, we selected a suitable threshold that could distinguish whether

122 position deviation occurs most accurately.

124 Cover Slip Misplacement

125 For a WSI with cover slip misplacement, an obvious black line appears. We use a method

126 based on template matching to generate a mask displaying the position of the cover slip. We

127 used the area threshold, the minimum area that we consider as the black region to adjust the

128 masks segmented from the thumbnails of the WSIs to judge whether cover slip misplacement

129 exists. In this study, we extended this method to obtain results that consist of classification

130 outcomes and position information. To obtain the results of cover slip misplacement, the

131 following steps are required:

132 1. We collected thumbnails of the WSIs from $I_{N}$ and $I_{C S M}$. 
2. We selected a thumbnail from $I_{C S M}$ obtained in step 1 and artificially drew the cover slip area as the template mask.

3. We generated masks through the template mask and corresponding features.

4. We removed the edge pieces of masks generated in step 3 and calculated the mean of each mask represented by a matrix obtained by computer technology. Then, we selected a suitable threshold to judge whether a WSI indicates cover slip misplacement.

141 is an example in which black areas on the border are detected on the border. Therefore, it is

142 necessary to remove the border pieces to exclude the effect of the existence of black areas on

143 the border. The masks are all binary images. The masks of WSIs with cover slip

144 misplacement contain more white pixels than masks of normal WSIs. Therefore, we chose a

145 threshold to judge whether WSIs have cover slip misplacement issues. In addition, the result

146 returned information on the position of the cover slip location to provide a reference for

147 correcting cover slip misplacement.

\section{Wrong Focus}

150 Figure 3 shows a comparison of a tile from a normal WSI and a WSI with wrong focus. We

151 could speculate that if a WSI is composed of tiles with lower information richness, it is more

152 likely to be a WSI with wrong focus. With the purpose of judging whether a WSI has wrong 153 focus, the following steps are required:

154 1. We collected thumbnails of WSIs from $I_{N}$ and $I_{W F}$. 

each WSI and then chose a suitable threshold to judge whether the WSIs have the wrong

173 focus issue.

\section{Results}

176 We designed experiments on two datasets to validate the effectiveness of the approach. We 
177 implemented the methods of each section and integrated these sections into an application.

\section{Training Set}

180

181 Position Deviation

182 An essential premise in this section is the accuracy of the classifier. We collected 1200 tiles,

183 including 900 for training and 300 for testing. The accuracy of the training set reaches 0.995 ,

184 and the accuracy of the testing set reaches 0.989 . Such accuracy is sufficient to guarantee the

185 proceeding of the left experiment in this section. An essential factor affecting the result is the

186 size of the tiles that we collected on the edge. Table 1 illustrates the effect of the tile size on

187 the results of this section.

188 Table 1. The relationship between the tile size and the performance of the method.

\begin{tabular}{|c|c|c|c|c|c|c|c|c|}
\hline & \multicolumn{2}{|c|}{ Tile_Size $=32$} & \multicolumn{2}{|c|}{ Tile_Size $=64$} & \multicolumn{2}{|c|}{ Tile_Size $=128$} & \multicolumn{2}{|c|}{ Tile_Size $=256$} \\
\hline & $\mathrm{N}$ & PD & $\mathrm{N}$ & PD & $\mathrm{N}$ & PD & $\mathrm{N}$ & PD \\
\hline \multicolumn{9}{|l|}{ Actual } \\
\hline $\mathrm{N}$ & 89 & 1 & 90 & 0 & 87 & 3 & 85 & 5 \\
\hline PD & 2 & 72 & 2 & 72 & 9 & 65 & 13 & 61 \\
\hline Accuracy & \multicolumn{2}{|c|}{0.982} & \multicolumn{2}{|c|}{0.988} & \multicolumn{2}{|c|}{0.927} & \multicolumn{2}{|c|}{0.890} \\
\hline Time & \multicolumn{2}{|c|}{2175} & \multicolumn{2}{|c|}{1726} & \multicolumn{2}{|c|}{1649} & \multicolumn{2}{|c|}{3013} \\
\hline
\end{tabular}

189 If the size of tiles is too large, then the accuracy will decrease because it may be more

190 possible that tiles we collected contain tissue areas. In contrast, if the size of tiles is too small,

191 then the accuracy may no longer ascend with a higher time consumption. The best value of

192 the tile size is 64.

193

194 Cover Slip Misplacement 
195 Due to the disturbance of the existence of other black areas, the selection of the area

196 threshold in segmenting the black line area from the thumbnail of the WSIs mentioned in the

197 Methods section is significant. Table 2 indicates the relationship between the area threshold

198 and the accuracy of judging whether WSIs have the cover slip misplacement issue.

199 Table 2. The relationship between the area threshold and the accuracy.

\begin{tabular}{cccccc}
\hline Area Threshold & 500 & 1000 & 2000 & 3000 & 5000 \\
Accuracy & 0.896 & 0.934 & 0.974 & 0.987 & 0.842 \\
\hline
\end{tabular}

200 If the value of the area threshold is too small, then the prediction may be affected by

201 other black areas of thumbnails. In contrast, if the value of the area threshold is too large,

202 then the actual cover slip misplacement issue could be ignored. The best area threshold is 2033000.

205 Wrong Focus

206 In this section, we experimented with three features, including the Brenner gradient function,

207 variance function and entropy function [22], compared with the Laplacian gradient function.

208 Table 3 illustrates the comparison of the accuracy using different features to judge whether

209 wrong focus exists in the WSIs.

210 Table 3. The contrast of the influence of different features on the prediction of wrong focus.

\begin{tabular}{lcc}
\hline Prediction of Different Features & Actual Normal & Actual Wrong Focus \\
\hline Brenner & 87 & 1 \\
Normal & 3 & 72 \\
Wrong Focus & 88 & 0 \\
Laplacian & 2 & 73 \\
Normal & & \\
Wrong Focus & 79 & 15 \\
Variance & 11 & 58
\end{tabular}


Entropy

$\begin{array}{lll}\text { Normal } & 84 & 14\end{array}$

$\begin{array}{lll}\text { Wrong Focus } & 6 & 59\end{array}$

212 Validation Set

213 This dataset includes 10 WSIs with position deviation, 17 WSIs with cover slip

214 misplacement, 24 WSIs with wrong focus and 20 normal WSIs. A WSI may have multiple

215 types of issues. Figure 4 illustrates the comparison between the results using the approach we

216 proposed in this work and the facts. In addition, the approach could produce a report to

217 provide results describing whether three issues exist and details related to existing issues.

218

219 Discussion

220 Previous studies have paid more attention to evaluating the quality of WSIs through clarity,

221 but they ignored the fact that other factors could also influence the quality. We proposed an

222 approach dealing with specific issues including the position deviation, cover slip

223 misplacement and wrong focus to evaluate the quality of WSIs and provided the details of

224 issues as references. We confirmed that issues could be discovered at a high accuracy. The

225 most significant consideration of this work is that we focus more on specific quality issues

226 rather than look for a single numeric value to represent the quality of WSIs. This work is the

227 first research study that regards the evaluation of WSIs as a project of discovering several

228 specific issues. This approach can be more meaningful than using only the clarity to represent

229 the quality of WSIs.

230 However, more work should be done. Limited specialized datasets with issues exist 
231 due to the lack of attention on the quality evaluation of WSIs. For instance, inappropriate

232 stain concentrations could affect the efficiency of clinical diagnostics. Previous studies

233 proposed some methods of evaluating the color reproducibility of WSIs [23-25]. However, it

234 is difficult to discover areas of improper stain concentrations in the WSIs. In addition,

235 methods of discovering areas with poor microtomy have not yet been proposed. Therefore,

236 we will collect WSIs with inappropriate stain concentrations and poor microtomy. In

237 addition, we will make an effort to develop methods to detect these issues.

239 Conclusions

240 In this paper, we proposed an approach to deal with several specific image issues including

241 position deviation, cover slip misplacement and wrong focus. The approach could discover

242 issues with high accuracy and produce reports providing details of the existing issues

243 discovered. However, the limitation of specialized datasets with issues and methods of

244 discovering such issues restrict the development of a quality evaluation. A dataset of WSIs

245 with more issues including stain concentration and poor microtomy should be established,

246 and methods discovering these issues should be developed in the future.

\section{List of Abbreviations}

249 WSI: whole-slide imaging; PD: position deviation; CSP: cover slip misplacement; WF:

250 wrong focus 
254 Ethics Approval and Consent to Participate

255 The present study was approved by the Ethics Committee of Guangdong Provincial People's 256 Hospital.

Consent for Publication

259 Written informed consent for publication was obtained for each participant.

Availability of Data and Materials

262 All data generated or analyzed during this study are included in this published article.

263

264 Competing Interests

265 The authors declare that they have no competing interests.

266

267 Funding

268 This work was supported in part by the Guangzhou People's Livelihood Science and

269 Technology Project under Grant 201803010097, in part by the Guangzhou City Industrial

270 Technology Major Research Project with Grant 201802010035, and in part by the Industrial

271 Internet Innovation and Development Project in 2018 Grant MIZ1824020-2.

272

273

Authors' Contributions

274 YS and YG designed the experiments and developed methods by coding. YS wrote the 
manuscript. CL and ZL collected the data required in the experiments and provided necessary background knowledge of the quality assessment of WSIs. All authors read and approved the

277 final manuscript.

278

Acknowledgements

280 Not applicable

281

282

\section{Authors'Information}

$283{ }^{1}$ School of Computer Science and Engineering, South China University of Technology,

284 Guangzhou, Guangdong 510006, China

$285{ }^{2}$ Department of Radiology, Guangdong Provincial People's Hospital, Guangdong Academy of 286 Medical Sciences, Guangzhou, Guangdong 510080, China

\section{References}

289 1. Al-Janabi S, Huisman A, Van Diest PJ. Digital pathology: current status and future perspectives. Histopathology. 2012;61:1-9.

291 2. Ghaznavi F, Evans A, Madabhushi A, Feldman M. Digital imaging in pathology: whole-slide imaging and beyond. Annu Rev Pathol. 2013;8:331-59.

293 3. Madabhushi A, Lee G. Image analysis and machine learning in digital pathology: challenges and opportunities. Med Image Anal. 2016;33:170-5.

4. Niazi MKK, Parwani AV, Gurcan MN. Digital pathology and artificial intelligence. 
297 5. Kayser K, Gortler J, Metze K, Goldmann T, Vollmer E, Mireskandari M, et al. How to measure image quality in tissue-based diagnosis (diagnostic surgical pathology).

299 Diagn Pathol. 2008;3:S11.

300 6. Zerbe N, Hufnagl P, Schluns K. Distributed computing in image analysis using open source frameworks and application to image sharpness assessment of histological whole slide images. Diagn Pathol. 2011;6:S16.

303 7. Shrestha P, Kneepkens R, van Elswijk G, Vrijnsen J, Ion R, Verhagen D, et al. Objective and subjective assessment of digital pathology image quality. AIMS Med Sci. $2015 ; 2: 65-78$.

8. Shrestha P, Kneepkens R, Vrijnsen J, Vossen D, Abels E, Hulsken B. A quantitative approach to evaluate image quality of whole slide imaging scanners. J Pathol Inform. 2016;7:56.

309 9. Walkowski S, Szymas J. Quality evaluation of virtual slides using methods based on comparing common image areas. Diagn Pathol. 2011;6:S14.

311 10. Hashimoto N, Bautista PA, Yamaguchi M, Ohyama N, Yagi Y. Referenceless image quality evaluation for whole slide imaging. J Pathol Inform. 2012;3:9.

313 11. Lopez XM, D'Andrea E, Barbot P, Bridoux AS, Rorive S, Salmon I, et al. An automated blur detection method for histological whole slide imaging. PLoS One. 2013;8:e82710. quality assessment for digital pathology. In: Lang K, Tingberg A, Timberg P, editors. Breast imaging - 13th international workshop, IWDM 2016, proceedings. Malmo, 
320 13. Jiménez A, Bueno G, Cristóbal G, Déniz O, Toomey D, Conway C. Image quality metrics applied to digital pathology. In: Schelkens P, Ebrahimi T, Cristóbal G, Truchetet F, Saarikko P, editors. Proceedings of SPIE 9896. Optics, photonics and digital technologies for imaging applications IV. Brussels, Belgium: SPIE; 2016. p. 118.

14. Campanella G, Rajanna AR, Corsale L, Schuffler PJ, Yagi Y, Fuchs TJ. Towards machine learned quality control: a benchmark for sharpness quantification in digital pathology. Comput Med Imaging Graph. 2018;65:142-51.

15. Ameisen D, Deroulers C, Perrier V, Bouhidel F, Battistella M, Legres L, et al. Towards better digital pathology workflows: programming libraries for high-speed sharpness assessment of whole slide images. Diagn Pathol. 2014;9:S3.

16. Janowczyk A, Zuo R, Gilmore H, Feldman M, Madabhushi A. HistoQC: an opensource quality control tool for digital pathology slides. JCO Clin Cancer Inform. 2019;3:1-7.

17. Pantanowitz L. Digital images and the future of digital pathology. J Pathol Inform. 2010;1:15.

18. Goode A, Gilbert B, Harkes J, Jukic D, Satyanarayanan M. OpenSlide: a vendorconvolutional neural networks. In: Advances in neural information processing systems. 2012. p. 1097-105. 
341 20. Harlick RM, Shanmugam K, Dinstein I. Textural features for image classification. IEEE Trans Syst Man Cybern. 1973;3:610-21.

343 21. Eskicioglu AM, Fisher PS. Image quality measures and their performance. IEEE Trans Commun. 1995;43:2959-65.

22. Sklansky J. Image segmentation and feature extraction. IEEE Trans Syst Man Cybern. $1978 ; 8: 237-47$.

23. Yagi Y. Color standardization and optimization in whole slide imaging. Diagn Pathol. 2011;6:S15.

24. Cheng WC, Keay T, O'Flaherty N, Wang J, Ivansky A, Gavrielides MA, et al.

25. Shrestha P, Hulsken B. Color accuracy and reproducibility in whole slide imaging scanners. J Med Imaging (Bellingham). 2014;1:027501.

Figure Legends

357 Figure 1. Examples of three issues claimed in this work. Figure 1(a) shows an example of

358 position deviation. Figure 1(b) shows an example of cover slip misplacement. Figure 1(c)

359 shows an example of wrong focus.

360 Figure 2. An example of a normal WSI that contains black areas on the border. Figure

361 2(a) is a normal thumbnail, and Figure 2(b) is a mask generated by the method. The white area on the border may influence the classification results. WSIs with white areas on the 
363 border may be wrongly classified as WSIs with cover slip misplacement. Therefore, it is

364 necessary to remove the edge of the original mask.

365 Figure 3. Comparison of pieces from the thumbnails of normal WSIs and WSIs with

366 Wrong Focus. Figure 3(a) shows a piece from a normal WSI thumbnail, and Figure 3(b)

367 shows a piece from a thumbnail of the WSI with wrong focus. It is apparent that pieces from

368 normal WSIs contain more useful information for clinic than pieces from WSIs with wrong

369 focus.

370 Figure 4. The contrast of the prediction provided by our approach and the facts. 


\section{Figures}

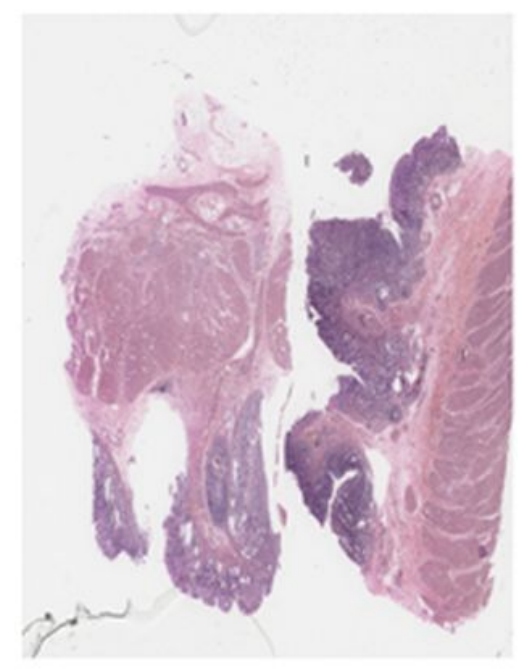

(a)

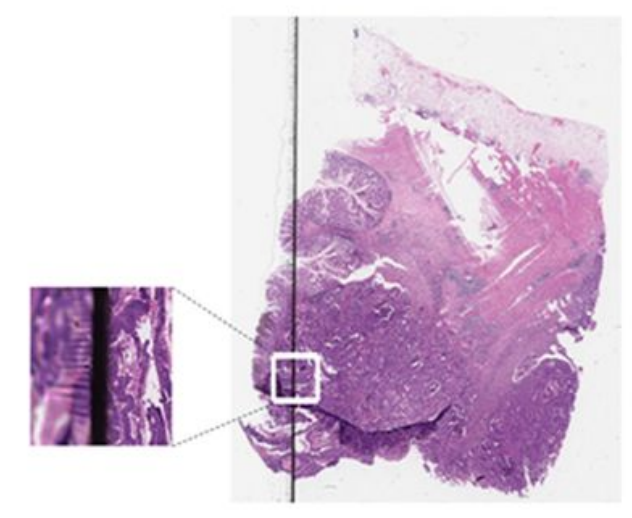

(b)

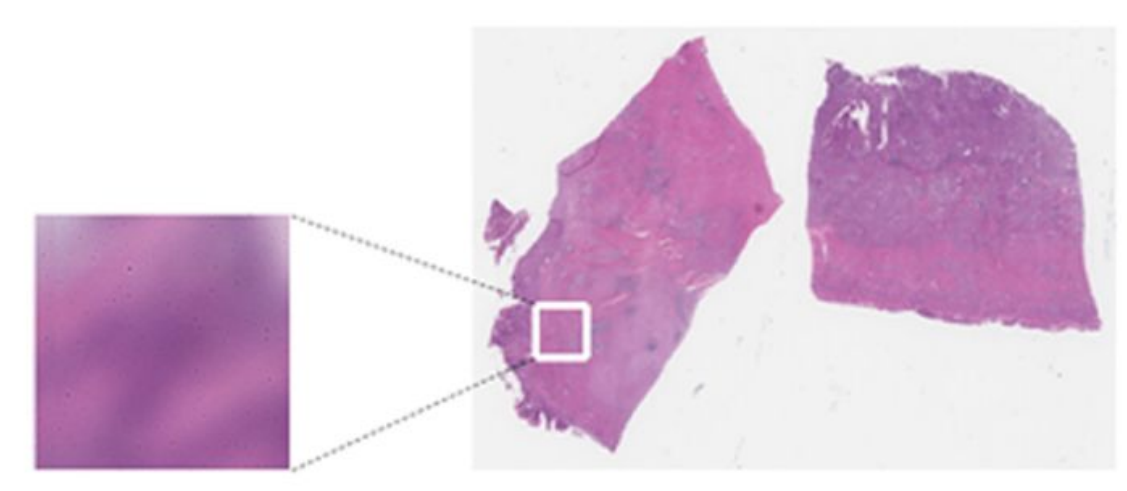

(c)

\section{Figure 1}

Examples of three issues claimed in this work. Figure 1(a) shows an example of position deviation. Figure 1(b) shows an example of cover slip misplacement. Figure 1(c) shows an example of wrong focus. 


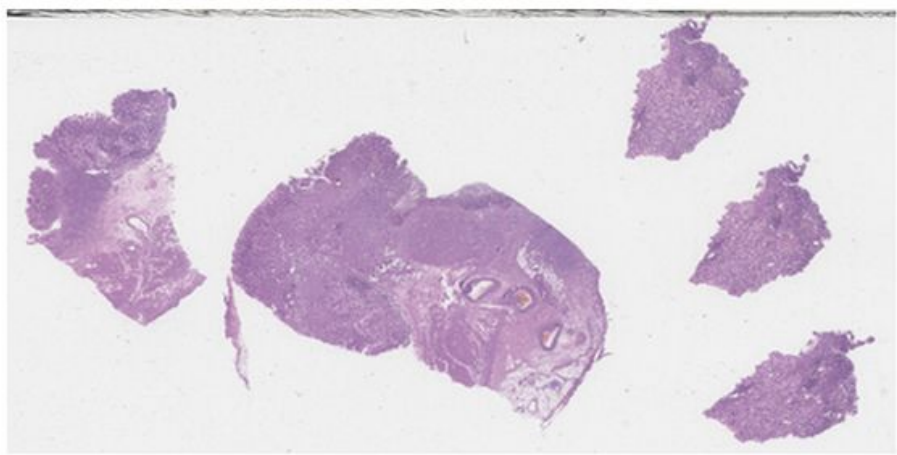

(a)

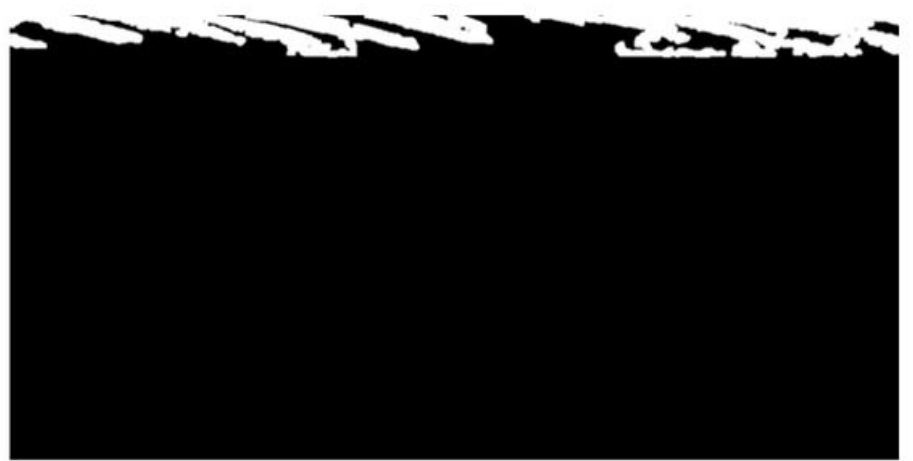

(b)

\section{Figure 2}

An example of a normal WSI that contains black areas on the border. Figure 2(a) is a normal thumbnail, and Figure 2(b) is a mask generated by the method. The white area on the border may influence the classification results. WSIs with white areas on the border may be wrongly classified as WSIs with cover slip misplacement. Therefore, it is necessary to remove the edge of the original mask.

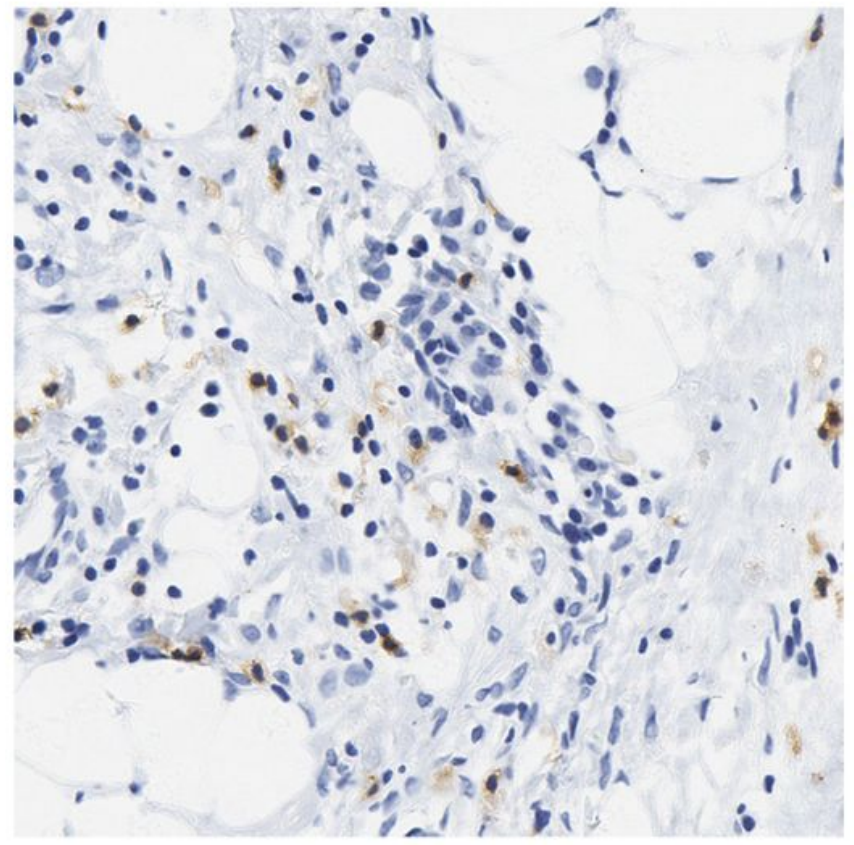

(a)

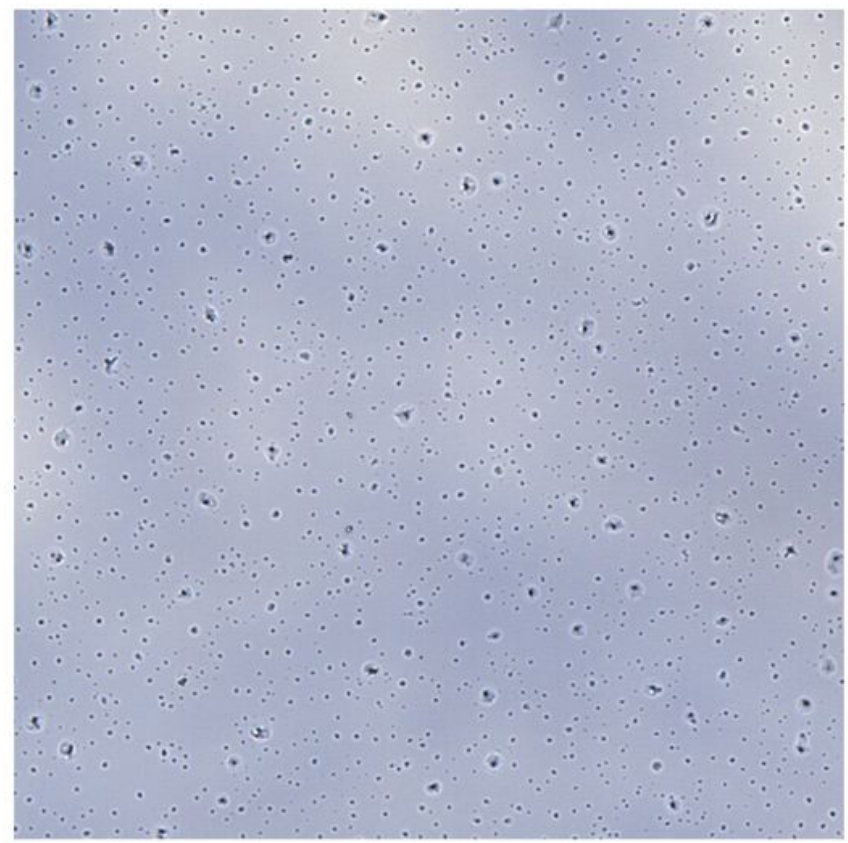

(b)

\section{Figure 3}

Comparison of pieces from the thumbnails of normal WSIs and WSIs with Wrong Focus. Figure 3(a) shows a piece from a normal WSI thumbnail, and Figure 3(b) shows a piece from a thumbnail of the WSI with wrong focus. It is apparent that pieces from normal WSIs contain more useful information for clinic than pieces from WSIs with wrong focus. 


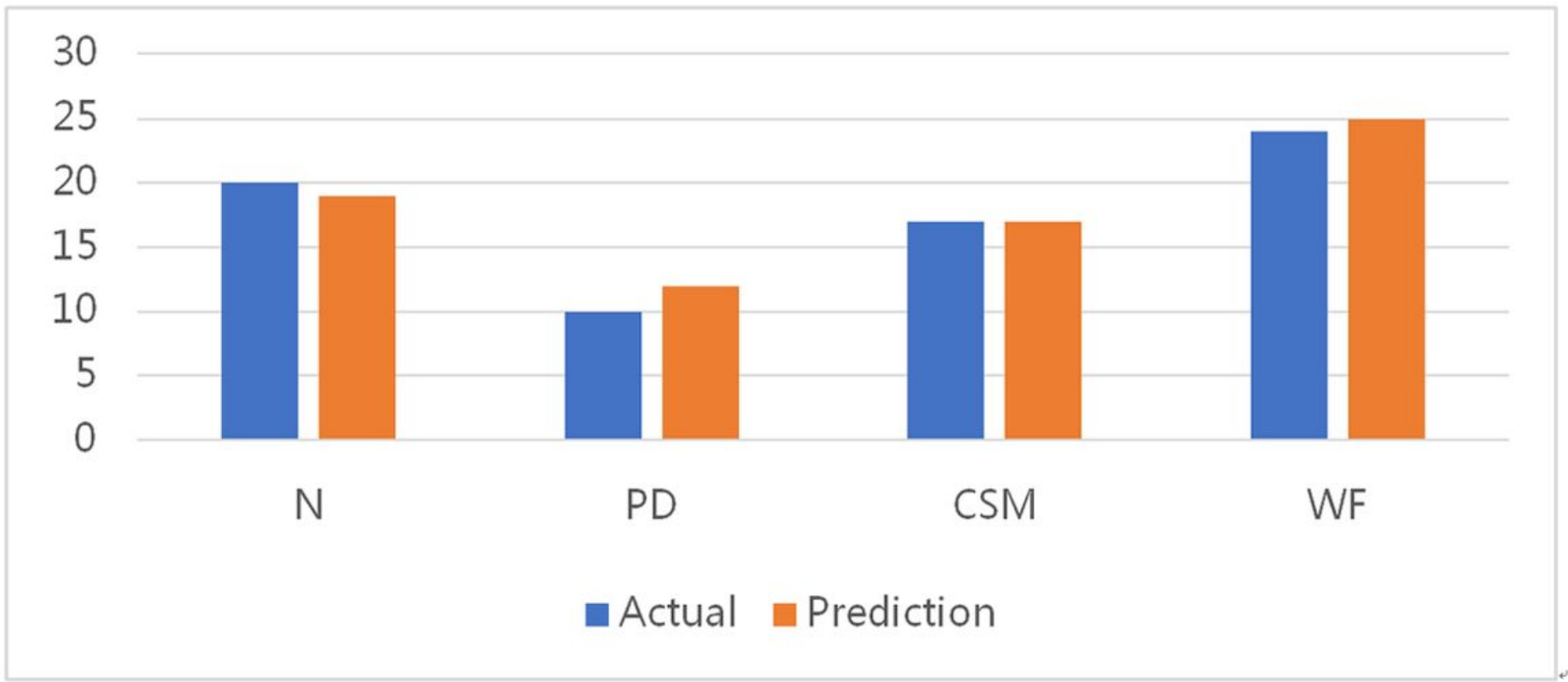

Figure 4

The contrast of the prediction provided by our approach and the facts. 